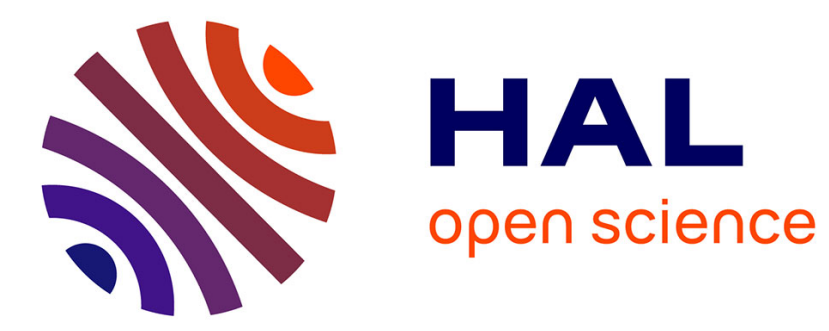

\title{
La profession réglementée du crédit
}

Thomas Puech

\section{To cite this version:}

Thomas Puech. La profession réglementée du crédit. Les professions (dé)réglementées - Bilans et perspectives juridiques, 2019. hal-03319993

\section{HAL Id: hal-03319993 \\ https://hal.science/hal-03319993}

Submitted on 5 Sep 2021

HAL is a multi-disciplinary open access archive for the deposit and dissemination of scientific research documents, whether they are published or not. The documents may come from teaching and research institutions in France or abroad, or from public or private research centers.
L'archive ouverte pluridisciplinaire HAL, est destinée au dépôt et à la diffusion de documents scientifiques de niveau recherche, publiés ou non, émanant des établissements d'enseignement et de recherche français ou étrangers, des laboratoires publics ou privés. 


\title{
LA PROFESSION RÉGLEMENTÉE DU CRÉDIT
}

\author{
Thomas PUECH \\ Doctorant contractuel à l'Université Toulouse 1 Capitole \\ (Institut de droit privé)
}

Depuis la loi $\mathrm{n}^{\circ}$ 41-2532 du 13 juin 1941 relative à la réglementation et à l'organisation de la profession bancaire ${ }^{1}$, la distribution du crédit fait l'objet de ce qu'il est coutumier de dénommer le "monopole bancaire". Le qualificatif de "monopole" est toutefois impropre ${ }^{2}$ en ce qu'il est ici usité non pas en vue de désigner une situation de marché où un acteur jouit d'une position dominante, mais une profession dont l'exercice "à titre habituel"3 nécessite - à peine de sévères sanctions ${ }^{4}$ - une autorisation préalable de l'État ${ }^{5}$. Il est également trompeur dans la mesure où, sous un vocable unique, sont visés, en vérité, trois "monopoles" distincts : le "monopole" de la réception de fonds remboursables du public, le "monopole" des services bancaires de paiement et le "monopole" des opérations de crédit ${ }^{6}$. Notre propos ayant pour objet d'étudier les incidences de la loi n ${ }^{\circ} 2015-990$ du 6 août 2015 pour la croissance, l'activité et l'égalité des chances économiques, dite loi Macron, quant à la seule profession du crédit, nous privilégierons en conséquence la dénomination plus exacte de "réglementation du crédit" dans les développements à venir, laquelle réglementation a donc pour effet de réserver l'octroi de crédit à titre habituel ${ }^{8}$ aux personnes dûment habilitées (les "professionnels du crédit”).

Traditionnellement justifiée par la volonté des autorités de contrôler et d'encadrer la distribution du crédit, clé de voûte de la politique monétaire ${ }^{9}$, cette réglementation tend aujourd'hui à être contestée ${ }^{10}$. En effet, sous l'influence conjuguée d'un contexte économique défavorable et d'un renforcement des contraintes prudentielles ${ }^{11}$, les professionnels du crédit se voient reprocher de ne plus exercer "leur métier consistant à prendre des risques pour prêter de l'argent à des entrepreneurs" "12. Nombreux sont donc les auteurs et praticiens qui appellent de leurs vœux une réforme de la réglementation actuelle du crédit afin d'en exempter les entités qui - à l'image des sociétés

\footnotetext{
${ }^{1}$ Elle fut complétée sous l'occupation par la loi nº 1941-06-14 du 14 juin 1941 relative à la réglementation et à l'organisation des professions se rattachant à la profession de banquiers, mais également à la libération par la loi $\mathrm{n}^{\circ} 45-15$ du 2 décembre 1945 relative à la nationalisation de la Banque de France et des grandes banques et à l'organisation du crédit.

${ }^{2}$ V. T. SAMIN, "Brèves réflexions sur le monopole bancaire (1984-2014)", Banque et droit, hors-série, mars 2014, p. 14.

${ }^{3}$ C. monét. fin., art. L. 511-5.

${ }^{4}$ Notamment, l'article L. 571-3 du Code monétaire et financier déclare passibles d'un emprisonnement de trois ans et d'une amende de 375000 euros, les personnes qui méconnaissent le monopole bancaire.

${ }^{5}$ Ladite autorisation prend la forme d'un agrément délivré par la Banque centrale européenne (BCE) ou l'Autorité de contrôle prudentiel et de résolution (ACPR) à l'issue d'une procédure décrite aux articles L. 511-9 et s. - tels que complétés par les articles R. 511-2-1 et s. - du Code monétaire et financier; S. NeuviLle, Droit de la banque et des marchés financiers, PUF, 2005 , p. $34, \mathrm{n}^{\circ} 16$.

${ }^{6}$ Ces trois activités répondent à la définition des “opérations de banque" visées à l'article L. 311-1 du Code monétaire et financier.

${ }^{7}$ A. Couret, J. Deveze et F. Peltier, Le droit bancaire, Que sais-je ?, PUF, 1994, p. 10, préfèrent, quant à eux, parler de "domaine réservé" des établissements de crédit sur l'exercice des opérations de banque.

${ }^{8}$ En application d'une jurisprudence constante, la condition d'habitude implique non seulement une succession d'opérations de crédit, mais également une pluralité de bénéficiaires : J. LASSERRE-CAPDEVILLE, "Les incidences sur le monopole bancaire et le monopole des prestataires de services de paiement de l'ordonnance sur le financement participatif", Gazette du Palais, $\mathrm{n}^{\circ}$ 26,18 sept. 2014, p. 5 et les références citées.

${ }^{9}$ La maîtrise du crédit demeure, en effet, un des principaux leviers de contrôle de la masse monétaire en circulation, comme le souligne M. RoussILle, "Que reste-t-il du monopole bancaire ?”, in Droit bancaire et financier, Mélanges AEBDF-France VI, Revue Banque éd., 2013, p. 615. Il est cependant reconnu que les évolutions contemporaines (fin de l'encadrement du crédit, recours aux nouveaux instruments de la politique monétaire et transfert de la conduite de celle-ci à la BCE) conduisent à affaiblir l'instrument de politique économique que constitue la distribution du crédit : Haut comité juridique de la place financière de Paris, Rapport sur le monopole bancaire, 14 mars 2016, p. 10.

${ }_{10}$ À titre d'exemple, voir le rapport réalisé en mai 2016 par le think tank Génération Libre et qui n'hésite pas à qualifier la réglementation du crédit de "rente" : Casser la rente bancaire française, consultable à partir du site generationlibre.eu.

${ }^{11}$ D. Lovato, Les normes prudentielles, Travaux de l'IFR (à paraître), Toulouse, 2016, spéc. p. 241-242.

${ }^{12}$ R. FERRAND, Assemblée nationale, Rapport fait au nom de la commission spéciale chargée d'examiner le projet de loi $\mathrm{n}^{\circ}$ 2447 pour la croissance et l'activité, t. 1, vol. 2, titres II à IV, compte rendu n ${ }^{\circ} 15$. Ce constat est partagé par une majorité de la doctrine: A. BordenAve, "L'été studieux du monopole bancaire", Hebdo édition affaires, n 437, 24 sept. 2015 ; J. LASSERRE-CAPDEVILLE, "La limitation du monopole bancaire par la reconnaissance du prêt inter-entreprises", RD banc. fin., $\mathrm{n}^{\circ}$ 1, janv. 2016, p. 3. D. LEGEAIS, "La loi Macron valide le prêt interentreprises", RTD Com., 2005, p. 565.
} 
de financement - n'accordent des crédits qu'à partir de leurs ressources propres ${ }^{13}$. Une telle réforme aurait d'ailleurs le mérite de rapprocher le droit français du droit communautaire, lequel n'élève le crédit au rang d'activité réservée qu'à condition que sa distribution soit couplée à la collecte de fonds remboursables du public ${ }^{14}$.

Fort de ce constat et afin de favoriser l'émergence de nouveaux acteurs susceptibles de pourvoir aux besoins de financement des entreprises, les pouvoirs publics tendent à multiplier les dérogations - que d'aucuns n'hésitent pas à qualifier de "brèches" 15 - à la réglementation actuelle du crédit, lesquelles se trouvent consacrées aux articles L. 511-6 et L. 511-7 du Code monétaire et financier selon - respectivement - qu'elles trouvent leur fondement dans la qualité des parties ou dans la nature des opérations ${ }^{16}$. La loi Macron, telle que complétée par un décret $n^{\circ}$ 2016-501 en date du 22 avril 2016, témoigne de cette volonté d'ouverture du marché du crédit en ce qu'elle autorise, entre autres mesure ${ }^{17}$ et sous conditions, les sociétés par actions et à responsabilité limitée ${ }^{18}$ à consentir des prêts d'argent à court terme à des microentreprises, des petites et moyennes entreprises (PME) ou des entreprises de taille intermédiaire (ETI) ${ }^{19}$, avec lesquelles elles entretiennent des liens économiques le justifiant ${ }^{20}$. L'innovation est certaine dans la mesure où, jusqu'à présent, un tel crédit n'était envisageable qu'entre sociétés ayant des liens capitalistiques ${ }^{21}$ ou contractuels ${ }^{22}$.

D’un point de vue économique, cette libéralisation - certes partielle - du marché du crédit a le mérite de favoriser le financement de l'économie réelle et de ne présenter que peu de risques en termes de politique monétaire, les sociétés exemptées ne pouvant octroyer des emprunts qu'à partir de leurs ressources disponibles.

D'un point de vue juridique, le juriste se trouve, d'ores et déjà, confronté à de multiples problématiques qui peuvent être résumées dans les termes suivants : quelles sont les exigences qui conditionnent le bénéfice du prêt inter-entreprises ? Aussi, quelle place est amené à prendre celui-ci au sein de la réglementation du crédit? Nous verrons que, soucieux de maintenir le financement inter-entreprises dans un cadre régulé et de préserver le "monopole bancaire", le législateur a procédé à une ouverture mesurée (I) et désordonnée (II) du marché du crédit.

\section{I - UNE OUVERTURE MESURÉE DU MARCHÉ DU CRÉDIT}

Le prêt inter-entreprises, et le crédit de manière générale, recèle de nombreux dangers. Tout d'abord, le prêteur s'expose au risque de ne jamais recouvrer le capital prêté, mais également de perdre un partenaire commercial crucial pour sa propre survie ${ }^{23}$. Ensuite, pèse sur l'emprunteur le risque de se soumettre à la domination économique et financière d'un créancier ${ }^{24}$. Enfin et comme nous le verrons a posteriori, ces risques sont exacerbés par l'application du droit commun du contrat de crédit. C'est pourquoi l'ouverture du marché du crédit ne s'est opérée qu'au prix de la mise en place de "garde-fous" 25 destinés à assurer la protection tant du prêteur que de l'emprunteur contre les dangers inhérents à ce type de financement. Est-ce à dire que l'ouverture est à la mesure desdits enjeux ? Nous le verrons en envisageant les exigences qui en conditionnent le bénéfice (A), mais également la maîtrise qu'ont entendu conserver les autorités au stade de sa mise en œuvre (B).

\footnotetext{
13 À titre d'exemple : A. Bordenave, "L'été studieux du monopole bancaire", op. cit. ; H. de Vauplane, "Le monopole bancaire sert-il (encore) à quelque chose ?", Revue Banque, 26 févr. 2015.

${ }^{14}$ H. de VAUPLANE, "Les premières brèches législatives au monopole bancaire", Revue Banque, n 765, nov. 2013, p. $20-21$.

${ }^{15}$ Ibid. ; H. de Vauplane, "La loi Macron et le crédit inter-entreprises : une nouvelle brèche dans le monopole bancaire", Les Echos, 15 févr. 2015.

${ }^{16}$ J. LASSERRE-CAPDEVILLE, "Les incidences sur le monopole bancaire et le monopole des prestataires de services de paiement de l'ordonnance sur le financement participatif', op. cit.

${ }^{17}$ Pour un exposé de celles-ci, voir T. GRANIER, "Financement des petites et moyennes entreprises", in La loi Macron du 6 août 2015 pour la croissance, l'activité et l'égalité des chances économiques, JCP E 2015, 1412, n 37.

${ }^{18} \mathrm{Il}$ en résulte que le prêteur ne peut qu'être une société anonyme (SA), une société en commandite par actions (SCA), une société par actions simplifiée (SAS), une société européenne (SE) ou une société à responsabilité limitée (SARL) : J. LASSERRECAPDEVILLE, "La limitation du monopole bancaire par la reconnaissance du prêt inter-entreprises", op. cit.

${ }^{19}$ Le législateur a donc restreint le bénéfice de l'exemption aux seules entreprises victimes - ou présumées l'être - de la pénurie de crédit bancaire. Du reste, il convient de rappeler que la détermination de la catégorie d'appartenance d'une entreprise microentreprise, PME ou ETI - s'opère par référence au décret $n^{\circ} 2008-1354$ du 18 décembre 2008.

${ }^{20}$ C. monét. fin., art. L. 511-6, $3^{\circ}$ bis, tel que modifié par l'article 167 de la loi.

${ }^{21}$ Crédit de trésorerie intra-groupe prévu par l'article L. 511-7, $3^{\circ}$ du Code monétaire et financier ; voir infra.

${ }^{22}$ Crédit fournisseur prévu par l'article L. $511-7,1^{\circ}$ du Code monétaire et financier ; voir infra.

${ }^{23}$ On peut parler d'effet domino en présence d'un tel risque de faillites en cascade... T. CharLes, "Délais de paiement et financement des entreprises", JCP E 2013, 393, n 22.

${ }^{24}$ J. LASSERRE-CAPDEVILLE, "La limitation du monopole bancaire par la reconnaissance du prêt inter-entreprises", op. cit.

${ }^{25}$ D. LEGEAIS, "La loi Macron valide le prêt interentreprises", op. cit.
} 


\section{A - Une ouverture conditionnée}

Le législateur a veillé à ce que l'accès au prêt inter-entreprises ne soit réservé qu'à des prêteurs disposant d'une assise financière solide (1) et agissant au profit de partenaires économiques (2).

\section{1) Le prêteur : une entreprise solide}

Comme nous l'avons vu en introduction de cette première partie, le prêt inter-entreprises expose le prêteur, outre le risque de non-remboursement, à la perte éventuelle d'un client important. C'est pourquoi le législateur et le gouvernement ont pris soin de restreindre le droit de prêter aux seules entreprises en mesure (ou à tout le moins présumées l'être...) d'encaisser les aléas naturels inhérents à toute activité de crédit. Il en résulte, en premier lieu, que les comptes de la société prêteuse doivent être certifiés par un commissaire aux comptes ${ }^{26}$. Il s'agit ainsi d'exclure "les prêteurs de taille modeste dont la solidité financière n'est pas encore avérée" 27 . Mais, dès lors que les SARL et les sociétés par actions qui - faute de dépasser les seuils - ne respectent pas cette exigence, peuvent néanmoins nommer volontairement et à cette fin un commissaire aux comptes, la pertinence de cette première restriction peut être contestée ${ }^{28}$.

En second lieu et de manière plus spécifique, le décret ajoute qu'à la date de clôture de chacun des deux exercices comptables précédant la date d'octroi du prêt, le prêteur doit disposer cumulativement (i) de capitaux propres supérieurs au montant de son capital social, (ii) d'un excédent brut d'exploitation positif et (iii) d'une trésorerie nette excédentaire ${ }^{29}$. En somme, "le prêt doit être octroyé sur des ressources propres et pérennes" ${ }^{30}$.

Ces restrictions au droit de prêter s'accompagnent, en outre, d'une limitation tenant à la qualité de l'emprunteur.

\section{2) L'emprunteur : un partenaire économique}

Dans l'esprit du législateur, la libéralisation du marché du crédit aux entreprises s'inscrit dans une logique de partenariat $^{31}$. Le crédit consenti en application de l'article 167 de la loi Macron a donc été envisagé tel un contrat d'intérêt commun, profitable aux deux parties. C'est pourquoi il ne peut être conclu qu'entre entreprises entretenant des liens économiques. La notion de "liens économiques", sujette à interprétation et conséquemment vectrice d'incertitude ${ }^{32}$, a été explicitée par décret ${ }^{33}$. Il est ainsi prévu que les liens doivent s'apprécier au niveau du groupe des sociétés parties au contrat ${ }^{34}$, le groupe étant ici défini par référence à l'article L. 233-16 du Code de commerce $^{35}$. En outre, le décret procède par énumération ; sans entrer dans le détail de celle-ci, il convient de retenir que les liens peuvent être de diverses natures : relation de sous-traitance, participation commune à un groupement d'intérêt économique, à un groupement attributaire d'un marché public ou à un projet subventionné, etc. Fort heureusement, l'énumération, très large, n'aboutit pas à un rétrécissement de la notion, mais bien plutôt à une interprétation extensive de celle-ci. Aussi, la prise en compte du groupe conduit à admettre que deux entreprises puissent se prêter mutuellement des fonds, en dépit de liens économiques seulement indirects ${ }^{36}$.

\footnotetext{
${ }^{26}$ C. monét. fin., art. L. 511-6, $3^{\circ}$ bis.

${ }^{27}$ B. GuilleminOT et J. Sutour, “L'extension du crédit interentreprise à un crédit partenaire”, Option finance, n ${ }^{\circ} 1337,19$ oct. 2015 , p. 27.

${ }^{28}$ A. BordenAVE, "L'été studieux du monopole bancaire", op. cit.

${ }^{29}$ C. monét. fin., art. R. $511-2-1-2,1^{\circ}$ et $2^{\circ}$.

${ }^{30}$ A. BORDENAVE, "Des coins toujours plus enfoncés dans le monopole bancaire", Hebdo édition affaires, n 465, 12 mai 2016.

${ }^{31} \mathrm{La}$ rédaction originelle du texte de loi (amendement $\mathrm{n}^{\circ} 1480$ ) allait même jusqu'à prévoir la formalisation de l'octroi du crédit au moyen d'un "contrat de partenariat" entre les sociétés.

${ }^{32}$ Sur les incertitudes qu'a suscité la notion avant la parution du décret, voir S. NEUVILLE, "Droit bancaire et financier - France ( $1^{\mathrm{er}}$ mars 2015 - 31 mars 2016)", Revue luxembourgeoise de bancassurfinance, 2016, n 02, chronique, p. 38.

${ }^{33}$ C. monét. fin., art. R. 511-2-1-1.

${ }^{34}$ A. BoRDENAVE, "Des coins toujours plus enfoncés dans le monopole bancaire", op. cit.

${ }^{35}$ C. monét. fin., art. R. 511-2-1-1, III : "Pour l'application du présent article et de l'article R. 511-2-1-2, le groupe s'entend de l'ensemble des entreprises entrant dans le même périmètre de consolidation au sens de l'article L. 233-16 du code de commerce lorsque l'organisation de la trésorerie de ces entreprises s'établit au niveau du groupe". Comme le souligne à juste titre B. BRIGNON, il est permis de se demander si la modification de l'article L. 233-16, IV du Code de commerce par l'ordonnance $\mathrm{n}^{\circ}$ 2015-900 du 23 juillet 2015 devra conduire à restreindre la définition du groupe retenue pour l'application du prêt Macron: "Chronique de droit des sociétés", Hebdo édition affaires, n 465, 12 mai 2016.

${ }^{36}$ T. BonNEAU, "Le régime du prêt interentreprises issu du décret du 22 avril 2016", Bulletin Joly Sociétés, $1^{\text {er }}$ juin 2016, n ${ }^{\circ}$ 06, p. 7.
} 
Ainsi liées, les entreprises sont en mesure de tirer des bénéfices divers de leur partenariat. S'agissant du prêteur, il est à même de mieux apprécier, évaluer et connaître le risque de crédit $^{37}$, mais également d'ajuster au mieux les montants prêtés aux besoins de financement et aux capacités de remboursement de son partenaire. Du côté de l'emprunteur, la nécessité d'un lien économique lui assure une convergence d'intérêts ${ }^{38}$ avec son créancier, convergence à même de faire naître une relation de confiance et d'éloigner - ne serait-ce que partiellement - le risque de dépendance.

En résumé et excepté l'obligation de certification des comptes sociaux, ces conditions nous semblent justifiées et légitimes en ce qu'elles aboutissent à une restriction du droit de prêter et du droit d'emprunter proportionnée au résultat escompté, à savoir répondre aux besoins ponctuels de financement des entreprises tout en s'assurant que les risques inhérents à ce type de crédit - et notamment le risque de faillites en cascade - soient limités. Une fois ces conditions remplies, le processus de financement peut donc être mis en œuvre et le contrat de crédit conclu. Toutefois, nous allons voir qu'en la matière, le législateur a entendu maintenir une emprise toute particulière sur le contenu contractuel.

\section{B - Une ouverture maîtrisée}

À défaut de stipulation contraire, le financement de l'emprunteur doit être soumis au régime du droit commun du contrat de crédit conclu entre professionnels (... et non pas professionnels du crédit), lequel se traduit par une liberté du taux de l'intérêt ${ }^{39}$, une formation retardée du contrat (contrat réel ${ }^{40}$ ) et un allègement des obligations et de la responsabilité du prêteur ${ }^{41}$. Ainsi présenté et en dépit de la convergence d'intérêts qui caractérise ce type de financement, le droit commun expose l'emprunteur aux dérives et abus (prêt usuraire, exonération de responsabilité en cas de soutien abusif ${ }^{42}$, etc.) et offre au prêteur une - trop ? - grande liberté d'action. Fort de ce constat et soucieux de réguler celle-ci, le législateur a pris le parti d'une libéralisation maîtrisée du crédit, en sorte que les modalités de conclusion et d'exécution du contrat ne sont pas laissées à la discrétion des parties. Cette maîtrise se manifeste au travers de conditions nombreuses et qu'il ne nous est pas paru opportun - au risque d'en travestir le sens - de regrouper. Elles seront donc évoquées unes à unes et tiennent au formalisme du prêt (1), à son montant (2), à sa durée (3), à son effet (4) et au sort de la créance de prêt (5).

\section{1) Le formalisme du prêt}

En premier lieu et dans un souci de transparence, l'opération de crédit doit être "formalisée"43 dans un contrat de prêt soumis au régime des conventions réglementées au sein de la société prêteuse ${ }^{44}$, ce qui signifie concrètement que sa conclusion sera subordonnée à l'autorisation préalable du conseil d'administration ou de l'assemblée des associés s'agissant respectivement d'une société par actions ${ }^{45}$ ou d'une SARL ${ }^{46}$. Aussi, le montant des prêts consentis doit être communiqué dans le rapport de gestion et faire l'objet d'une attestation du commissaire aux comptes. Plus précisément et en application de l'article R. 511-2-1-3 du Code monétaire et financier, ce dernier

\footnotetext{
${ }^{37}$ N. AuClAir, "La possibilité de prêts interentreprises au sein de la loi Macron : une occasion manquée", Gazette du Palais, 24 mai $2016, \mathrm{n}^{\circ} 19$, p. 58.

${ }^{38}$ B. Dondero et C. TABourot-Hyest, "Le crédit interentreprises est désormais opérationnel”, JCP E 2016, 443, nº 21.

${ }^{39}$ C. monét. fin., art. L. 313-5.

${ }^{40}$ D. LEGEAIS, "La loi Macron valide le prêt interentreprises", op. cit.

${ }^{41}$ J. LASSERRE-CAPDEVILLE, "La limitation du monopole bancaire par la reconnaissance du prêt inter-entreprises", op. cit. ; N. AuClair, op. cit. ; D. LeGEAIS, "Le crédit interentreprises réactivé", RD banc. fin., $\mathrm{n}^{\circ}$ 5, sept. 2015, repère 5.

${ }^{42}$ Article L. 650-1 du Code de commerce.

${ }^{43}$ La loi a sans doute voulu dire un écrit : H. CAUSSE, "Loi Macron : dispositions de droit bancaire", Hebdo édition affaires, $\mathrm{n}^{\circ}$ 434, 3 septembre 2015.

${ }^{44}$ Certains commentateurs ont cru bon d'étendre cette exigence à la société emprunteuse : B. DONDERO, "La loi Macron : autres mesures de droit des sociétés", Bulletin Joly Sociétés, $\mathrm{n}^{\circ}$ 10, p. 546. Il est vrai que les travaux parlementaires - et notamment l'amendement présenté par D. SASSONE-ESTROSI devant le Sénat le 9 avril 2015 - pouvaient être invoqués à l'appui de cette lecture extensive de l'article L. 511-6, $3^{\circ}$ bis. Cependant, dans la mesure où la lettre des articles L. 511-5, $3^{\circ}$ bis et R. 511-2-1$3 \mathrm{du}$ Code monétaire et financier ne visent que les prêts "consentis", il semble qu'une interprétation extensive des personnes visées par la procédure des conventions réglementées soit à proscrire.

${ }^{45}$ Article L. 511-6, $3^{\circ}$ bis, qui renvoie aux articles L. 225-38 à L. 225-40 du Code de commerce. Comme l'ont souligné à juste titre de nombreux auteurs, les dispositions relatives aux SA à directoire et conseil de surveillance, aux SE, aux SCA et aux SAS ne sont pas visées par les textes ; est-ce à dire que les prêts consentis par celles-ci pourraient valablement échapper à la procédure des conventions réglementées ? Le doute est permis, mais une réponse négative semble tout de même s'imposer dans la mesure où est ici en cause la protection des intérêts du prêteur : B. BRIGNON, op. cit.

${ }^{46}$ Articles L. 223-19 et L. 223-20 du Code de commerce.
} 
est avisé annuellement des prêts en cours consentis par le prêteur et doit attester, dans une déclaration jointe au rapport de gestion et pour chaque contrat, (i) du montant initial, (ii) du capital restant dû par l'emprunteur et (iii) du respect des dispositions qui régissent lesdits prêts. Ainsi présentée, l'intervention du commissaire aux comptes apparaît à la fois "peu efficace et lourde" ${ }^{47}$ : peu efficace dans la mesure où il n'est amené à intervenir qu'a posteriori, une fois le crédit consenti et - le cas échéant - le risque pris ; lourde dans la mesure où il ne peut se contenter d'une attestation globale portant sur l'ensemble des prêts consentis au cours de l'exercice, mais doit rentrer dans le détail de chaque contrat.

\section{2) Le montant du prêt}

En deuxième lieu, le montant du prêt se trouve doublement plafonné. D'une part et en vertu de l'article R. 5112-1-2, $3^{\circ}$, le montant annuel des prêts consentis par une même entreprise ne doit pas dépasser $50 \%$ de sa trésorerie nette (ou $10 \%$ de la trésorerie nette consolidée de son groupe si elle appartient à un groupe de sociétés), sans pouvoir excéder en toute hypothèse un plafond fixé en fonction de sa catégorie d'appartenance, à savoir 10 millions d'euros pour une PME, 50 millions d'euros pour une ETI et 100 millions d'euros pour une grande entreprise. Il s'agit là d'une application large et quantitative du caractère accessoire que doivent revêtir les opérations de crédit pour l'entreprise prêteuse ${ }^{48}$.

D'autre part, vient s'ajouter à ce plafond annuel général un plafond annuel spécial. En effet, l'article R. 5112-1-2, $4^{\circ}$, énonce que le montant annuel de l'ensemble des prêts accordés par une même entreprise à une autre entreprise ne peut être supérieur au plus grand des deux montants suivants, à savoir $5 \%$ de la trésorerie nette ou, dans la limite de 10000 euros, $25 \%$ du plafond fixé en fonction de la catégorie d'appartenance du prêteur. L'objectif est d'éviter que le prêteur ne "mette tous ses œufs dans le même panier"49, et donc ne concentre le risque de crédit sur un seul et même débiteur ; il s'agit également d'éviter que ce dernier ne se place, d'un point de vue financier, sous la domination excessive - pour ne pas dire abusive... - du premier ${ }^{50}$. Cependant, force est de reconnaître que les seuils édictés paraissent bien trop restrictifs ; notamment, la limite annuelle de 10000 euros semble bien dérisoire en considération des besoins de financement de nombreuses PME et ETI ${ }^{51}$.

\section{3) La durée du prêt}

En troisième lieu, la durée du financement fait elle aussi l'objet d'un encadrement dans la mesure où elle ne peut excéder deux ans ${ }^{52}$. Faute de précisions supplémentaires, se pose la question de la licéité d'un prêt renouvelé par une clause de tacite reconduction? A priori et dans le silence des textes, un tel renouvellement semble possible (il sera dès lors constitutif d'un nouveau contrat ${ }^{53}$ ), à condition toutefois que les exigences conditionnant le bénéfice du prêt inter-entreprises soient à nouveau respectées... ${ }^{54}$

\section{4) L'effet du prêt}

En quatrième lieu, il est prévu aux articles L. 511-6, $3^{\circ}$ bis et R. 511-2-1-1, III du Code monétaire et financier que l'octroi d'un prêt ne peut avoir pour effet de placer l'emprunteur dans un état de dépendance économique contraire aux dispositions du second alinéa de l'article L. 420-2 du Code de commerce ${ }^{55}$ et, spécialement, de lui imposer des délais de paiement ne respectant pas les plafonds légaux définis aux articles L. 441-6 et L. 443-1 du code de commerce ${ }^{56}$. Il s'agit là d'une solution de bon sens et qui a vocation à ce que la finalité première du prêt - à savoir l'assistance portée à un partenaire économique en quête de financement - ne soit dévoyée en vue de

\footnotetext{
47 N. AUCLAIR, op. cit.

${ }^{48}$ A. BordenAVE, "Des coins toujours plus enfoncés dans le monopole bancaire", op. cit.

49 T. BONNEAU, op. cit.

${ }^{50}$ A. Masounave, “PME : le crédit interentreprises, une fausse bonne idée ?”, Revue Banque, n 782,26 févr. 2015.

${ }^{51}$ Paris EUROPLACE, Financement en Dette des PME/ETI : Nouvelles Recommandations, mars 2014, rapport consultable à l'adresse suivante : http://www.paris-europlace.net/files/Rapport_PEP_PME_100314.pdf

${ }^{52}$ C. monét. fin., art. L. 511-6, $3^{\circ}$ bis.

${ }^{53}$ Y. Guenzoui et C. Hannoun, Terme, Répertoire de droit civil, n 31, oct. 2008 (actualisation : juin 2016).

54 N. AuClair, op. cit. ; B. BRIGNON, op. cit.

55 "Est en outre prohibée, dès lors qu'elle est susceptible d'affecter le fonctionnement ou la structure de la concurrence, l'exploitation abusive par une entreprise ou un groupe d'entreprises de l'état de dépendance économique dans lequel se trouve à son égard une entreprise cliente ou fournisseur. Ces abus peuvent notamment consister en refus de vente, en ventes liées, en pratiques discriminatoires visées au I de l'article L. 442-6 ou en accords de gamme".

${ }^{56}$ H. CAUSSE, op. cit.
} 
contourner les règles élémentaires du droit de la concurrence. On peut d'ailleurs penser que le nouvel article 1143 du Code civil ${ }^{57}$ concourra également et utilement au maintien d'un certain équilibre entre les parties.

\section{5) Le sort de la créance de prêt}

En cinquième et dernier lieu, l'article L. 511-6, $3^{\circ}$ bis énonce que "nonobstant toute disposition ou stipulation contraire, les créances détenues par le prêteur ne peuvent, à peine de nullité, être acquises par un organisme de titrisation [...] ou un fonds professionnel spécialisé [...] ou faire l'objet de contrats constituant des instruments financiers à terme ou transférant des risques d'assurance à ces mêmes organismes ou fonds". Le législateur a donc voulu prévenir toute financiarisation par voie de titrisation du prêt inter-entreprises ${ }^{58}$, financiarisation qui serait contraire à la logique de partenariat prônée par les textes, notamment en ce qu'elle pourrait aboutir à mettre l'emprunteur en lien avec un prêteur - cessionnaire de la créance de prêt le cas échéant - inconnu. Néanmoins et dans le silence des textes, rien ne semble interdire la cession de la créance de prêt à des entités non visées par le texte, un établissement de crédit par exemple ${ }^{59}$.

Au terme de ces développements, le constat est le suivant : il est vrai que la liberté d'action des parties devait être encadrée, d'autant plus en une matière où l'application du droit commun du contrat de crédit aurait potentiellement été source d'abus et de dérives. Des ajustements étaient donc nécessaires afin de tempérer les envies de prêt et les velléités d'emprunt, et ainsi limiter les prises de risques de part et d'autre. N'est-on pour autant pas allé trop loin, notamment au regard des caractéristiques traditionnellement recherchées de ce type de financement par les acteurs du marché (souplesse, rapidité, efficacité ${ }^{60}$ ) ? Nous pensons, pour l'essentiel, aux conditions tenant au formalisme, au montant et à la durée du prêt, lesquelles nous semblent par trop restrictives ${ }^{61}$. Du reste, n'aurait-il pas été préférable, entre partenaires, de mettre l'accent sur l'intuitus personae qui caractérise le contrat de prêt ? Ainsi et dans le prolongement du devoir de bonne foi consacré à l'article 1104 du Code civil, aurait pu être utilement consacré un devoir de collaboration obligeant les parties à coopérer tout au long du contrat $^{62}$. Notamment, le professionnel-prêteur qui, à la différence d'un professionnel du crédit, ne dispose que de moyens techniques limités de mesure et de suivi du risque de crédit ${ }^{63}$, aurait pu trouver dans un pareil devoir le moyen d'obtenir une information - a priori mais également a posteriori - sur l'évolution de la situation économique et financière de l'emprunteur. Cette information lui aurait permis de mieux apprécier l'étendue du risque pris tout en enrichissant le processus de décision présidant à l'octroi du crédit. Cela est d'autant plus vrai dans un contexte où la transparence des comptes sociaux tend à être restreinte par le législateur lui-même, ne serait-ce qu'au travers de l'article 213 de la loi Macron ${ }^{64}$.

Cela dit, il convient dorénavant de se pencher sur la méthode de la réforme, laquelle suscite des réserves dans la mesure où elle aboutit à une libéralisation désordonnée du marché du crédit.

\section{II - UNE OUVERTURE DESORDONNÉE DU MARCHÉ DU CRÉDIT}

La réglementation du crédit admet de multiples tempéraments qui figurent aux articles L. 511-6 et L. 511-7 du Code monétaire et financier ${ }^{65}$ et dont l'articulation avec le prêt inter-entreprises peut être source de difficultés. Plus précisément, nous tâcherons de mettre en évidence la superposition confuse de mécanismes de financement qui en est la conséquence (A) et qui aboutit à un enchevêtrement incohérent de corps de règles distincts (B).

\footnotetext{
57 "Il y a également violence lorsqu'une partie, abusant de l'état de dépendance dans lequel se trouve son cocontractant, obtient de lui un engagement qu'il n'aurait pas souscrit en l'absence d'une telle contrainte et en tire un avantage manifestement excessif"; G. CHANTEPIE et M. LATINA, La réforme du droit des obligations, commentaire théorique et pratique dans l'ordre du Code civil, Dalloz, 2016, p. 280, n 340.

${ }^{58}$ T. GRANIER, op. cit.

${ }^{59}$ J. LASSERRE-CAPDEVILLE, "La limitation du monopole bancaire par la reconnaissance du prêt inter-entreprises", op. cit.

${ }^{60}$ S. de FAY, "Finance de l'innovation, innovation(s) dans la finance", in Étude en partenariat avec le Centre de recherche LegalEdhec, JCP E 2016, 1480, n 37.

${ }^{61}$ H. Le NABASQue, "Prêt inter-entreprises : le décret est paru !", RD banc. fin., $\mathrm{n}^{\circ}$ 3, mai 2016, repère 3.

${ }^{62}$ C'est le sens du propos de D. LegEAIs qui s'interroge sur l'opportunité d'un droit du prêteur : D. LegEAIs, "Le crédit interentreprises réactivé", op. cit.

${ }^{63}$ Haut comité juridique de la place financière de Paris, op. cit., p. 11.

${ }^{64}$ A. del Pozo, "Le nouvel univers de crédit crée des opportunités", Option finance, 9 nov. 2015.

${ }^{65}$ S. NeUVILLE, op. cit., p. $271, \mathrm{n}^{\circ} 174$.
} 


\section{A - Un désordre consécutif à la multiplicité des crédits inter-entreprises}

La réglementation du crédit comporte, comme nous venons de l'indiquer, de nombreux assouplissements tendant à favoriser le financement des entreprises. Or, trois d'entre eux semblent pouvoir entrer en conflit avec le prêt Macron $^{66}$ : il s'agit du crédit de trésorerie intra-groupe (1), des délais et avances de paiement (2) et de la souscription de titres de créance (3).

\section{1) Le prêt inter-entreprises à l'épreuve du crédit de trésorerie intra-groupe}

En application de l'article L. 511-7, I, $3^{\circ}$ du Code monétaire et financier, la réglementation du crédit ne fait pas “obstacle à ce qu'une entreprise, quelle que soit sa nature, puisse procéder à des opérations de trésorerie avec des sociétés ayant avec elle, directement ou indirectement, des liens de capital conférant à l'une des entreprises liées un pouvoir de contrôle effectif sur les autres". Dans une première approche de cette disposition, deux séries de questions trouvent à se poser : qu'est-ce qu'une "opération de trésorerie" ? Que doit-on entendre par "contrôle effectif" ? Loin de vouloir revenir sur des questions déjà longuement débattues en doctrine ${ }^{67}$, retenons simplement que, pour ce qui intéresse notre étude, l'article L. 511-7, I, $3^{\circ}$ doit être interprété largement comme autorisant toute entreprise à octroyer un crédit au bénéfice d'une entreprise du même groupe sur laquelle elle exerce un contrôle effectif, notion de fait abandonnée à l'appréciation souveraine des juges du fond.

Du point de vue de leur nature et dans la mesure où elles sont synonymes d'opérations de crédit, les opérations de trésorerie dépassent le simple cadre du contrat de prêt ${ }^{68}$. Un enchevêtrement de ces deux dispositifs de financement peut donc se produire en présence d'un prêt accordé par une entreprise au bénéfice d'un partenaire économique membre du même groupe de sociétés. Conscient de cette difficulté, le Gouvernement a tenu à préciser que la mise en œuvre du prêt Macron ne devait pas être confondue avec celle du crédit de trésorerie intra-groupe ${ }^{69}$. Il en résulte que ces derniers peuvent coexister harmonieusement et ainsi enrichir la palette des financements nonbancaires accessibles aux entreprises.

\section{2) Le prêt inter-entreprises à l'épreuve des délais et avances de paiement}

Aussi, l'article L. 511-7, I, $1^{\circ}$ du Code monétaire et financier énonce qu'en dépit de la réglementation du crédit, les entreprises, quelle que soit leur nature, peuvent "dans l'exercice de leur activité professionnelle consentir à leurs contractants des délais ou avances de paiement". À première vue, aucune confusion n'est envisageable : en effet, les délais et avances de paiement constituent des opérations de crédit mais qui, cette fois-ci, ne peuvent juridiquement prendre la forme d'un contrat de prêt ${ }^{70}$. Lorsqu'ils sont accordés en faveur d'entreprises partenaires $^{71}$, ils ne peuvent donc être assimilés au prêt inter-entreprises ici étudié, ce que confirme a fortiori la lettre de l'article R. 511-2-1-1, III du Code monétaire et financier.

Il faut néanmoins tenir compte de la position des juges qui, de manière constante et sur le fondement de l'article L. 511-7, I, $1^{\circ}$, admettent que les entreprises peuvent valablement consentir des prêts à leurs contractants dès lors que lesdits prêts sont liés à leur activité commerciale et constituent le complément indissociable d'un contrat commercial $^{72}$. Cette interprétation extensive - pour ne pas dire audacieuse ${ }^{73}$ - des termes de la loi contribue, en conséquence, à obscurcir les frontières apparemment claires qui séparent le délai (ou l'avance) de paiement du

\footnotetext{
${ }^{66}$ A. Bordenave, "Des coins toujours plus enfoncés dans le monopole bancaire", op. cit.

${ }^{67}$ T. BonNEAU, Droit bancaire, LGDJ, 2015, p. 196 et s., $n^{\circ} 277$ et les références citées.

${ }^{68}$ Aux termes de l'article L. 313-1 du Code monétaire et financier, "constitue une opération de crédit tout acte par lequel une personne agissant à titre onéreux met ou promet de mettre des fonds à la disposition d'une autre personne ou prend, dans l'intérêt de celle-ci, un engagement par signature tel qu'un aval, un cautionnement, ou une garantie. Sont assimilés à des opérations de crédit le crédit-bail, et, de manière générale, toute opération de location assortie d'une option d'achat". Le domaine des opérations de crédit est donc plus vaste que celui du contrat de prêt, dans la mesure où il comprend - outre la mise à disposition de fonds à titre onéreux - le crédit par signature ou encore le crédit-bail.

${ }^{69}$ C. monét. fin., art. R. 511-2-1-1, III.

${ }^{70}$ D. LEGEAIS, "La loi Macron valide le prêt interentreprises", op. cit.

${ }^{71}$ Sur ce point, remarquons que les facilités de paiement peuvent être accordées à tout contractant de l'entreprise, et non pas seulement aux entreprises avec lesquelles elle est en relation d'affaires : S. ALAMOWITCH, "Nouveau regard sur une exception négligée au monopole bancaire", RD banc. fin., $\mathrm{n}^{\circ}$ 5, sept. 2014, prat. 6.

${ }^{72}$ CA Paris, $5^{\mathrm{e}}$ ch. B, 22 mai 2003 ; CA Colmar, $1^{\text {re }}$ civ., sect. B, 29 juin 2005 ; CA Bordeaux, $2^{\mathrm{e}}$ ch. civ., 26 févr. 2015 , $\mathrm{n}^{\circ}$ 12/05363; CA Poitiers, $2^{\mathrm{e}}$ ch. civ., 7 avr. 2009.

${ }^{73} \mathrm{~L}$ 'audace des juges peut être tempérée au regard de la position des autorités, et notamment d'une lettre interprétative adressée le 6 décembre 1985 par le Directeur du Trésor au Président du Conseil National du Patronat Français, laquelle indiquait déjà que le législateur n'avait pas entendu interdire d'une manière générale la distribution de crédits qui sont étroitement liées à l'activité commerciale de l'entreprise et qui constituent le complément indissociable d'un contrat commercial.
} 
prêt. Dans le silence des textes, le sort de cette jurisprudence fait aujourd'hui débat et laisse entier le problème de son articulation avec le prêt inter-entreprises consacré par la loi Macron...

\section{3) Le prêt inter-entreprises à l'épreuve de la souscription de titres de créance}

Enfin, l'article L. 511-7, I, $4^{\circ}$ autorise les entreprises de toute nature à émettre des titres, qu'il s'agisse de titres financiers ou de bons de caisse. Ces titres, dans la mesure où ils peuvent conduire à une avance temporaire de fonds au bénéfice de l'émetteur, sont un instrument privilégié du crédit inter-entreprises. Aussi et bien que l'article précité ne vise que la seule "émission" de titres, c'est-à-dire la réception de fonds pour ce qui concerne notre étude, il faut comprendre par là même que la souscription de titres, c'est-à-dire l'avance des fonds, est également autorisée ${ }^{74}$.

Tout comme en matière de délais et avances de paiement, la frontière avec le contrat de prêt - et, le cas échéant, le prêt inter-entreprises - est, en apparence, clairement délimitée : tout d'abord parce qu'il est constant - tant en doctrine qu'en jurisprudence - qu'un titre de crédit n'obéit pas à une même nature, et donc à un même régime juridique, que le contrat de prêt ; ensuite parce que le législateur a pris soin, une nouvelle fois, de préciser que leur périmètre respectif ne devait pas être confondu ${ }^{75}$.

Toutefois, ce premier regard porté sur la distinction entre le titre de crédit et le contrat de prêt ne résiste pas à l'analyse. Ainsi et à titre d'exemple, qu'est-ce qu'un bon de caisse, si ce n'est un titre causé par un contrat de prêt, c'est-à-dire ni plus ni moins que la reconnaissance d'une dette d'emprunt aux yeux de nombreux auteurs ${ }^{76}$ ! De même, la doctrine pointe les difficultés mises en lumière par les "EuroPP", instruments de financement interentreprises pouvant prendre la forme - selon les cas - d'un contrat ou d'un titre, sans que l'on puisse, dans les faits, réellement les distinguer ${ }^{77}$. Enfin et sans prétendre à l'exhaustivité, remarquons que les frontières entre l'investissement et le prêt sont encore plus ténues en matière de financement participatif, où de nombreux auteurs pointent l'illusoire distinction opérée par le législateur entre le "crowdequity" - entendons le financement au moyen de la souscription de titres - et le "crowdlending" - entendons le financement au moyen de la conclusion d'un contrat de prêt ${ }^{78}$.

Cette présentation sommaire des difficultés d'articulation pouvant naître de la coexistence d'une pluralité de mécanismes de financement démontre que le domaine du prêt inter-entreprises demeure incertain. De fait, on peut regretter que le législateur ait fait fi des exceptions préexistantes lors de l'élaboration de la loi Macron, même s'il

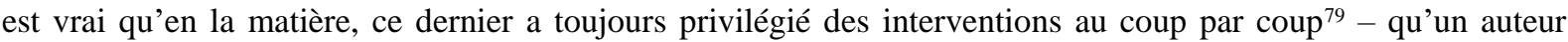

\footnotetext{
${ }^{74}$ T. SAMIN, op. cit., p. 15.

${ }^{75}$ C. monét. fin., art. R. 511-2-1-1, III.

${ }^{76}$ H. Hovasse, N. Martial-Braz et S. le Normand-CAillere, Bons de caisse, Fasc. JurisClasseur, n 1950, 30 nov. 2013.

${ }^{77}$ Haut comité juridique de la place financière de Paris, op. cit., p. 22-23 ; A.-R. Cuny De LA VerRyere, "Le crédit semiobligataire : un instrument de financement d'origine allemande adapté aux PME françaises", Bulletin Joly Bourse, $1^{\mathrm{er}}$ mars $2013, n^{\circ} 03$, p. 152.

78 Notamment, V. Perruchot-Triboulet, "L'encadrement juridique du prêt opéré par le biais d'une plateforme de financement participatif", Bulletin Joly Sociétés, $\mathrm{n}^{\circ}$ 12, p. 756, qui estime qu'en pratique, "le financement d'une entreprise ce n'est pas crédit ou souscription au capital mais bien plutôt crédit et souscription au capital. Il est surprenant que le législateur n'ait pas souhaité faire le lien entre ces deux univers qui sont un peu présentés et réglementés de façon distincte dans le Code monétaire et financier sans que des passerelles aient été jetées entre ces différentes modalités de financement [...]. C'est dommage que cette occasion n'ait pas été saisie pour avancer un peu sur la question de l'hybridation et du mélange des genres qui s'opère de plus en plus sur la tête de l'investisseur dont on ne sait plus toujours s'il est associé ou prêteur de deniers" (nous soulignons).

79 À titre d'illustration: décret $\mathrm{n}^{\circ}$ 2013-717 du 2 août 2013 modifiant certaines règles d'investissement des entreprises d'assurance ; charte relative aux "Euro Private Placements" ou "Euro PP" de mars 2014 ; ordonnance n 2014-559 du 30 mai 2014 relative au financement participatif ; décret $n^{\circ} 2014-1530$ du 17 décembre 2014 modifiant les règles d'investissements des entreprises d'assurances, des institutions de prévoyance, des mutuelles et de leurs unions dans les prêts à l'économie ou les titres assimilés ; règlement (UE) n ${ }^{\circ} 2015 / 760$ du 29 avril 2015 relatif aux fonds européens d'investissement à long terme ; loi $\mathrm{n}^{\circ} 2015-992 \mathrm{du} 17$ août 2015 relative à la transition énergétique pour la croissance verte ; ordonnance $\mathrm{n}^{\circ} 2016-520$ du 28 avril 2016 relative aux bons de caisse ; décret $\mathrm{n}^{\circ} 2016-1272$ du 29 septembre 2016 relatif aux investissements participatifs dans les projets de production d'énergie renouvelable ; décret $\mathrm{n}^{\circ} 2016-1453$ du 28 octobre 2016 relatif aux titres et aux prêts proposés dans le cadre du financement participatif ; décret $n^{\circ} 2016-1587$ du 24 novembre 2016 fixant les conditions dans lesquelles certains fonds d'investissement peuvent octroyer des prêts aux entreprises.
} 
n'hésite pas à qualifier de "méthode des petits pas" ${ }^{80}$ - au détriment d'une refonte globale. Il en résulte un manque de lisibilité et d'accessibilité du prêt inter-entreprises ${ }^{81}$ qui se prolonge naturellement au stade de son régime par une superposition incohérente de régimes distincts.

\section{B - Un désordre constitutif de distorsions de concurrence}

Faute de réforme globale, les retouches successives apportées à la réglementation du crédit, et spécialement à la réglementation du crédit inter-entreprises, ont aboutit à la coexistence de mécanismes concurrents de financement. La superposition de multiples corps de règles qui en est la conséquence est source de difficultés dans la mesure où une activité de même nature - la distribution de crédit(s) le cas échéant - se trouve soumise, selon les cas, à un régime juridique distinct. En pareille hypothèse et pour reprendre la terminologie du professeur BERGEL, on peut parler non pas de "différence" de régime mais bien plutôt de "discrimination"82. Dans le prolongement des développements précédents, notre propos mérite d'être illustré. À titre d'exemple, il est difficilement compréhensible qu'une entreprise puisse librement prêter des fonds à une autre entreprise dès lors que cette dernière est sous le contrôle de la première (opération de trésorerie intra-groupe), alors que l'octroi d'un même prêt à un partenaire économique suppose le respect de conditions draconiennes. De même et concernant le prêt d'argent découvert par la jurisprudence sur le fondement de l'article L. 511-7, I, $1^{\circ}$, on peut estimer que sa survie est incertaine ; notamment, on voit mal comment les juges pourraient faire fi de l'encadrement légal dont fait aujourd'hui l'objet le prêt inter-entreprises en vue de perpétuer la jurisprudence précitée ${ }^{83}$. Autrement dit, la consécration d'une exception légale en faveur du financement des entreprises risque d'aboutir, dans les faits, à une limitation des opportunités de financement puisque, dans le prolongement de l'article L. 511-7, I, $1^{\circ}$ précité, le prêt d'argent prétorien pouvait être accordé par une entreprise au profit d'un contractant quelconque dès lors que - pour rappel - ledit prêt constituait le complément d'un contrat commercial et participait de son activité commerciale, à l'exclusion de toute autre condition. Enfin, c'est en matière de souscription de titres que les incohérences sont les plus manifestes. Ainsi, comment expliquer que le financement d'une même entreprise par une société agissant à titre accessoire emporte l'application de règles différentes touchant au taux de l'intérêt ou encore au montant du prêt selon que celui-ci s'opère via la souscription de "minibons" (titres dérivés des bons de caisse) ou la conclusion d'un contrat ${ }^{84}$ ? Il ne faut dès lors pas s'étonner que de nombreux auteurs et praticiens pointent du doigt ces discriminations qui, dans les faits, se traduisent par de graves distorsions de concurrence ${ }^{85}$. Celles-ci sont d'autant plus graves lorsqu'est en cause la protection de l'emprunteur : en effet, longtemps considéré comme la "partie faible" au contrat, les règles qui assurent la défense de ses intérêts se trouvent aujourd'hui éclatées... C'est pourquoi un même emprunteur, selon qu'il s'adresse à tel prêteur-professionnel du crédit ou à tel autre prêteur-professionnel, ne bénéficie pas des mêmes droits ${ }^{86}$.

Qui plus est, ces discriminations favorisent l'adoption de comportements opportunistes par les acteurs de marché : en effet, conscients de la coexistence de multiples corps de règles, ils n'hésitent pas à opérer des arbitrages entre les divers pans de la réglementation du crédit. Ainsi et pour ne retenir que le seul exemple du financement participatif, nombreuses sont les plateformes à reconnaître que le choix de leur statut - intermédiaire en financement participatif (IFP) ou conseiller en investissement participatif (CIP) - ne s'est pas opéré en fonction de la nature des opérations - contrat ou titre de prêt - intermédiées, mais en fonction du régime applicable à celles$\mathrm{ci}^{87}$. C'est d'ailleurs de tels comportements qui ont conduit le Gouvernement à procéder à la réforme des bons de

\footnotetext{
${ }^{80}$ A. Bordenave, "L'été studieux du monopole bancaire", op. cit. ; H. de VAuPLANE, "Monopole bancaire : la loi de transition énergétique ouvre une nouvelle brèche", Les Echos, 5 oct. 2015.

${ }^{81}$ Haut comité juridique de la place financière de Paris, op. cit., p. 10.

82 J.-L. BERGEL, "Différence de nature (égale) différence de régime", RTD civ., 1984, p. 255.

${ }^{83}$ N. AuClaIR, op. cit. ; Haut comité juridique de la place financière de Paris, op. cit., p. 19.

${ }^{84}$ H. de VAuPLANE, "Le financement des entreprises par la blockchain : le cas des minibons", RTD financier, n ${ }^{\circ}$ 2, 2016, p. 64.

${ }^{85}$ M. Roussille, op. cit., p. 607 et s., spéc. p. 616-619; Haut comité juridique de la place financière de Paris, op. cit., p. 19 ; B. BENEDICTE, "Quelle banque est en train de naître ? La banque bionique ?", Gazette du Palais, 10 nov. 2015, n 314 , p. 3. En matière de financement participatif, voir l'avis du Comité consultatif du secteur financier (CCSF) en date du 14 avril 2014 ; A.-V. LE FuR, "Enfin un cadre juridique pour le crowdfunding, une première étape dans la réglementation", Recueil Dalloz, 2014, p. 1831.

${ }^{86}$ D. LEGEAIS, "La fin annoncée du monopole bancaire”, RD banc. fin., $\mathrm{n}^{\circ} 2$, mars 2015, repère 2 ; D. LEGEAIS, "La loi Macron valide le prêt interentreprises", $o p$. cit., p. 565, lequel s'interroge : "à quoi bon avoir introduit autant de règles protectrices s'il devient aussi facile de les contourner?".

${ }^{87} \mathrm{~F}$. LACROIX, qui évoque l'attitude de certaines plateformes ayant eu pour objectif de contourner les plafonds légaux imposés par la loi pour certains prêts à intérêt accordés par des personnes physiques agissant dans un cadre non-professionnel : "Plateformes de crowdfunding : quel statut pour quelles opérations ?", Bulletin Joly Bourse, $1^{\text {er }}$ juin 2015, n 6, p. 253.
} 
caisse et à la création des minibons par l'ordonnance $\mathrm{n}^{\circ} 2016-520 \mathrm{du} 28$ avril 2016... ${ }^{88}$ (minibons dont on a vu, de manière paradoxale, qu'ils étaient eux-aussi source de discriminations, à moins que le législateur n'ait volontairement décidé de combattre le mal par le mal...).

Fort de ces précisions, la réglementation du crédit, et spécialement la réglementation du crédit aux entreprises, apparaît fracturée et incohérente. Les règles qui en constituent le socle sont à géométrie variable ; elles mériteraient d'être améliorées, ne serait-ce que pour satisfaire aux objectifs de lisibilité et d'intelligibilité de la loi ${ }^{89}$. Une réforme globale de la réglementation est donc souhaitable, mais encore faut-il s'entendre sur les solutions envisageables. Nous croyons qu'une telle réforme devrait s'appuyer sur la création d'un droit commun de l'activité de crédit, qui comporterait un socle de règles applicables à toute personne habilitée à prêter des fonds ${ }^{90}$. Ce droit commun pourrait ensuite reprendre l'actuelle distinction entre les professionnels du crédit et les simples professionnels ${ }^{91}$, tout en harmonisant les régimes applicables à chacun d'eux ${ }^{92}$. Ce n'est qu'à ce prix que la réglementation française du crédit pourrait retrouver les qualités requises d'accessibilité et d'attractivité, mais aussi sa légitimité. L’avenir de la profession réglementée du crédit en dépend...

${ }^{88}$ R. VABRES, “Bons de caisse, minibons, blockchain... résurrection ou révolution ?”, Droit des sociétés, $\mathrm{n}^{\circ}$ 7, juill. 2016, repère 7.

${ }^{89}$ A. Pietranscosta, "Le monopole bancaire et ses justifications - éléments de réflexion", in Rapport sur le monopole bancaire, 14 mars 2016, p. 31 et 41.

${ }^{90}$ Haut comité juridique de la place financière de Paris, op. cit., p. 24, qui évoque la nécessité d'ériger un "tronc commun de régulation".

${ }^{91}$ On pense notamment aux seuils de l'usure, aux plafonds d'emprunt et aux critères de l'activité de crédit (critère de l'accessoire, de l'habitude, de la profession, etc.).

${ }^{92}$ A. Pietranscosta, op. cit., p. 43, évoque le souhait d'une distinction nouvelle entre le "prêteur qualifié" et "l'emprunteur qualifié". 